\title{
Absence of Breakdown of Continuous Symmetry in Two-dimensional Models of Statistical Physics
}

\author{
R. L. Dobrushin and S. B. Shlosman \\ Institute for Problems of Information-Transmission, Moscow, USSR
}

Received July 19, 1974

\begin{abstract}
Two-dimensional lattice model is considered. The connected Lie group $G$ acts on a configuration space. The Gibbs potential assumed to be invariant under this action. We prove, that under general assumption on the potential, each Gibbs random field with this potential is also $G$ invariant.
\end{abstract}

\section{Introduction}

There exist numerous examples of breakdown of discrete symmetry in spin models of statistical physics. Among them we can enumerate the Ising model $[1,2]$, the anisotropic Heisenberg model [3] etc. In all these examples there is an action of a disconnected group on a configuration space, and the Gibbs potential remains invariant. However, the Gibbs random fields with this potential turn out to be non-invariant under this action.

The goal of the present paper is to show that if the group $G$ is a compact connected Lie group and the field is two-dimensional, the situation is quite opposite. Namely, under some general assumptions, $G$-invariance of the potential implies $G$-invariance of each Gibbs random field with this potential.

\section{Main Result}

Let $\mathbb{Z}^{2}$ be a two-dimensional lattice with points $t=\left(k_{1}, k_{2}\right)$, where $k_{i}$ are integers. Let $G$ be a compact connected Lie group, which acts (on the left) on a space $X$. In other words, there is a mapping $\pi: G \times X \rightarrow X$ with the property $\pi\left(g_{2}, \pi\left(g_{1}, x\right)\right)=\pi\left(g_{2} g_{1}, x\right)$, where $g_{1}, g_{2} \in G, x \in X$.

Let $\mu$ be a $G$-invariant $\sigma$-finite measure on $X$, defined on a $G$-invariant $\sigma$-algebra $\mathscr{B}_{X}$ of subsets of $X$. Let us fix a structure of Riemannian manifold and a two-side invariant Riemannian metric on $G$. Let $\mathscr{B}_{G}$ be $\sigma$-algebra of Borel subsets of $G$. The action of $G$ on $X$ is assumed to be measurable, i.e. the mapping $\pi: G \times X \rightarrow X$ is measurable. (In the space $G \times X$ the $\sigma$-algebra $\mathscr{B}_{G} \times \mathscr{B}_{X}$ is considered.)

Let $A \subseteq \mathbb{Z}^{2}$ be any subset. Configuration on $A$ is an arbitrary function $x_{A}: A \rightarrow X$; it is determined by its values $x_{A}=\left(x_{t} ; t \in A\right), x_{t} \in X$. Denote $X^{A}$ the set of all configurations on $A$. If $J \subset A$, then $x_{J}=\left(x_{t} ; t \in J\right)$ means restriction $\left.x_{A}\right|_{J}$. On the set of all configurations on $A$ a measure $\mu_{A}$ is naturally introduced as a product of $|A|$ measures $\mu$. (Here and further on $|A|$ denotes the cardinality of $A$.) $\mu_{A}$ is 
defined on the $\sigma$-algebra $\mathscr{B}_{X^{A}}$ - the product of $|A| \sigma$-algebras $\mathscr{B}_{X}$. On the set of all configurations $X^{A}$ the group $G$ acts in a natural way, by:

$$
\left(g x_{A}\right)_{t}=g x_{t} ; \quad t \in A, \quad x_{A} \in X^{A}, \quad g \in G .
$$

A potential $U$ is a system of functions $\left\{U_{A} ; A \subset \mathbb{Z}^{2},|A|<\infty\right\}$, where every function $U_{A}=U_{A}\left(x_{A}\right), x_{A} \in X^{A}$ is defined on $X^{A}$. Let the following conditions on potential be satisfied.

A. Invariance. For any $A \subset \mathbb{Z}^{2},|A|<\infty$

$$
U_{A}\left(g x_{A}\right)=U_{A}\left(x_{A}\right) ; \quad g \in G, \quad x_{A} \in X^{A} .
$$

B. Differentiability. Let $A \subset \mathbb{Z}^{2},|A| \geqq 2, t_{1}, t_{2} \in A, t_{1} \neq t_{2}$ and $x_{A} \in X^{A}$. Let us consider a certain subset of configurations arising from $x_{A}$ under the following action of the group $G \times G$ on $X^{A}$ :

$$
\left[\left(g_{1}, g_{2}\right) * x_{A}\right]_{t}= \begin{cases}g_{1} x_{t_{1}} & t=t_{1} \\ g_{2} x_{t_{2}} & t=t_{2} \\ x_{t} & t \neq t_{1}, t_{2} .\end{cases}
$$

Then on the group $G \times G$ we can define the function

$$
V_{A, t_{1}, t_{2}, x_{A}}\left(g_{1}, g_{2}\right)=U\left[\left(g_{1}, g_{2}\right) * x_{A}\right] \text {. }
$$

On the group $G \times G$ we have a two-side invariant Riemannian metric. Let $w_{1}, w_{2}$ be any pair of left-invariant unit vector fields on $G \times G$. There should exist and be uniformly restricted second derivatives

$$
w_{1}\left[w_{2}\left(V_{A, t_{1}, t_{2}, x_{A}}\left(g_{1}, g_{2}\right)\right)\right] \leqq L
$$

for any finite $A$, any $x_{A} \in X^{A}$ and any $t_{1}, t_{2}$.

If we introduce the system of local coordinates $y_{1}, \ldots, y_{2 n}$ on $G \times G$ in such a way that the vector fields $\partial / \partial y_{k}$ were left invariant and of unit length, our condition (1.2) can be rewritten in a more familiar manner

$$
\frac{\partial}{\partial y_{k}} \frac{\partial}{\partial y_{l}} V_{A, t_{1}, t_{2}, x_{A}}\left(y_{1}, \ldots, y_{2 n}\right) \leqq L \quad[4,5] \text {. }
$$

C. Finiteness. There exist a number $d_{0}, 1 \leqq d_{0}<\infty$ such that

$$
U_{A}\left(x_{A}\right) \equiv 0,
$$

when $d(A)>d_{0}$, where $d(A)$ is the diameter of $A$.

D. Convergence and Measurability. Let $A \subset \mathbb{Z}^{2}$ be finite and $J \subseteq A$. The function $U_{A}\left(x_{A}\right)$ is assumed to be $\mathscr{B}_{X^{A}}$-measurable and the integral

$$
\int_{X^{J}} \exp \left\{-U_{A}\left(x_{J}, x_{A \backslash J}\right)\right\} \mu_{J}\left(d x_{J}\right)
$$

converge for any $A, J \subseteq A$ and $x_{A \backslash J} \in X^{A \backslash J}$.

Notes. It follows from conditions $\boldsymbol{A}$ and $\boldsymbol{B}$ that the potential $U$ is continuous on any orbit of $G$ action on $X$. Hence, if the action is transitive, the condition $\boldsymbol{D}$ follows from the remaining ones. 
Let $J \subset \mathbb{Z}^{2}$ be a finite subset, $\bar{J}$ be its complement. A configuration $x_{\bar{J}} \in X^{\bar{J}}$ will be called a boundary condition for volume $J$. Define the energy of the configuration $x_{J} \in X^{J}$ under the boundary condition $x_{\bar{J}}$ by

$$
U\left(x_{J} \mid x_{\bar{J}}\right)=\sum_{A: A \cap J \neq \emptyset} U_{A}\left(x_{A}\right),
$$

where for any $A$, the restrictions of $x_{A}$ satisfy the following equalities:

$$
\left.x_{A}\right|_{A \cap J}=\left.x_{J}\right|_{A \cap J} ;\left.\quad x_{A}\right|_{A \cap \bar{J}}=\left.x_{\bar{J}}\right|_{A \cap \bar{J}} .
$$

The conditional Gibbs density in the volume $J$ with boundary conditions $x_{\bar{J}}$ and potential $U$ is defined by

$$
P\left(x_{J} \mid x_{\bar{J}}\right)=\frac{\exp \left\{-U\left(x_{J} \mid x_{\bar{J}}\right)\right\}}{\int_{X^{J}} \exp \left\{-U\left(x_{J} \mid x_{\bar{J}}\right)\right\} \mu_{J}\left(d x_{J}\right)} .
$$

The existence of the integral in the above formula is consequence of conditions $\boldsymbol{C}$ and $\boldsymbol{D}$ on the potential.

In the configuration space $X^{\mathbb{Z}^{2}}$, let us introduce the $\sigma$-algebra $\mathfrak{A}$, generated by the cylindrical sets of the form $\mathscr{D}\left(B_{t}, t \in J\right)=\left\{\left(x_{t}, t \in \mathbb{Z}^{2}\right) \in X^{\mathbb{Z}^{2}} ; x_{t} \in B_{t}, t \in J\right\}$ where $J \subset \mathbb{Z}^{2}$ is finite, and $B_{t} \in \mathscr{B}_{X}$ for any $t \in J$. By "state" of the system we mean a probability measure $\mathscr{P}$ on the space $\left(X^{\mathbb{Z}^{2}}, \mathfrak{A}\right)$. For any $I \subseteq \mathbb{Z}^{2}$, let us denote by $\mathfrak{A}_{I}$ the $\sigma$-algebra, generated by $\mathscr{D}\left(B_{t}, t \in J\right)$, where $J \subseteq I$ is finite.

A Gibbs state [6-8] with potential $U$, is defined as a state such that for any finite $J \subset \mathbb{Z}^{2}$ and any $B_{t} \in \mathscr{B}_{X}, t \in J$, the conditional probability of the event $\mathscr{D}\left(B_{t}, t \in J\right)$ with respect to $\sigma$-algebra $\mathfrak{A}_{J}$ is the function of $y_{\mathbb{Z}^{2}} \in X^{\mathbb{Z}^{2}}$ of the form

$$
\mathscr{P}\left\{\mathscr{D}\left(B_{t}, t \in J\right) \mid \mathfrak{A}_{\bar{J}}\right\}=\int_{\prod_{t \in J} B_{t}} P\left(x_{J} \mid y_{\bar{J}}\right) \mu_{J}\left(d x_{J}\right),
$$

where $y_{\bar{J}}=\left.y_{\mathbb{Z}^{2}}\right|_{\bar{J}}$. It follows from this definition the existence of mutual densities (or correlation functions in terms of statistical physics) $P\left(x_{J}\right)$ which satisfy the following condition:

$$
\mathscr{P}\left(\mathscr{D}\left(B_{t}, t \in J\right)\right)=\int_{\prod_{t \in J} B_{t}} P\left(x_{J}\right) \mu_{J}\left(d x_{J}\right) .
$$

E.g., we can choose them according to the formula:

$$
P\left(x_{J}\right)=\int_{\mathbb{Z}^{2}} P\left(x_{J} \mid y_{\bar{J}}\right) \mathscr{P}\left(d y_{\mathbb{Z}^{2}}\right) .
$$

The main result of this paper is the following

Theorem. If the potential $U$ satisfies assumptions $\boldsymbol{A}-\boldsymbol{D}$, then every Gibbs state $\mathscr{P}$ with this potential is a G-invariant measure on $\left(X^{\mathbb{Z}^{2}}, \mathfrak{A}\right)$ with respect to the natural action (1.1) of $G$ on $X^{\mathbb{Z}^{2}}$.

This theorem should be interpreted as the illustration of an opinion, spread among physicists, that no breakdown of continuous symmetry occurs in twodimensional systems. The assumption about the two-dimensional nature of the lattice is of great importance, which corresponds to common physical ideas, stating that the breakdown of continuous symmetry is possible for three-dimen- 
sional lattices. The assumption about the existence of second derivatives of the potential, is also essential. It's natural to expect, that without this assumption, our theorem is not true. (See discussions in paragraph 6 of this article.) On the other hand, the supposition about the finiteness of the potential is introduced to simplify the proofs. It seems possible to extend our method on potentials, which decrease rapidly enough at infinity.

Our conditions include a model in which $X=\mathbb{R}^{3}$ is the three dimensional euclidean space, $G=S O(3)$ is the group of proper rotations, $\mu$ is a $G$-invariant measure on $\mathbb{R}^{3}$ with finite support;

$$
U_{A}\left(x_{A}\right)= \begin{cases}-k\left\langle x_{t}, x_{t^{\prime}}\right\rangle & \text { for } A=\left\{t, t^{\prime}\right\},\left|t-t^{\prime}\right|=1 ; \\ 0 & \text { for other } A .\end{cases}
$$

Here, $\langle$,$\rangle denote a usual scalar product. In particular, if the measure \mu$ is concentrated on the sphere $S^{2} \subset \mathbb{R}^{3}$, our model reduces to the classical Heisenberg model. This allows us to generalize, to some extent, the results by Mermin [9] about the absence of spontaneous magnetisation in two-dimensional Heisenberg model. It is necessary to compare our results with results by Fisher, Jasnow [10], and Garrison et al. [11], having the same physical interpretation, but expressed in other terms and obtained by different methods, based on the Bogolubov inequality. On the other hand, the method of our article is based on a local limit theorem of probability theory for non-identicaly distributed random variables. As a matter of fact, this method presents mathematical interpretation of traditional physical intuition (see for example Fisher [12].

\section{Main Lemma}

To prove the main theorem, it is important to consider at first the case, when our group $G$ is a circle $S^{1}$ with standard smooth structure.

Let $V_{n}, n=1,2, \ldots$ be a square $V_{n}=\left\{t=\left(k_{1}, k_{2}\right) \in \mathbb{Z}^{2} ;-n \leqq k_{1}, k_{2} \leqq n\right\}$. For any $I, J \subset I$ and any boundary condition $x_{\bar{I}}$ let us consider mutual densities

$$
P_{J, I}\left(x_{J} \mid x_{\bar{I}}\right)=\int_{X \backslash J J} P\left(x^{I} \mid x_{\bar{I}}\right) \mu_{I \backslash J}\left(d x_{I \backslash J}\right),
$$

$x_{I}=\left(x_{J}, x_{I \backslash J}\right)$. Let $m_{S}$ be the Haar measure on $S^{1}\left(m_{S}\left(S^{1}\right)=1\right)$.

If a group $H$ acts on the left on a space $Y$, denote $H\left(y_{0}\right)$ the orbit (under $H$ ) of the point $y_{0} \in Y$, and, for any function $f: H\left(y_{0}\right) \rightarrow \mathbb{R}^{1}, f^{H}\left(\cdot, y_{0}\right)$ is the function on $H$, defined by $f^{H}\left(h, y_{0}\right)=f\left(h y_{0}\right)$.

Let $x_{V_{k}} \in X^{V_{k}}$ and $S^{1}\left(x_{V_{k}}\right) \subset X^{V_{k}}$ be an orbit of $x_{V_{k}}$ under $S^{1}$-action on $X^{V_{k}}$ (see 1.1). Let's consider $P_{V_{k}, V_{n}}$ as a function on this orbit.

Lemma 1. For any $\varepsilon>0$ and $k \geqq 0$ there exist a constant $c_{k}$, such that for any $n \geqq k$, any boundary condition $x_{\overline{V_{n}}} \in X^{\overline{V_{n}}}$, any $x_{V_{k}}$ and any $g_{0} \in S^{1}$ we have

$$
\begin{array}{r}
\int_{S^{1}}\left|P_{V_{k}, V_{n}}^{S^{1}}\left(b_{1}, x_{V_{k}} \mid x_{\overline{V_{n}}}\right)-P_{V_{k}, V_{n}}^{S^{1}}\left(g_{0} b_{1}, x_{V_{k}} \mid x_{\overline{V_{n}}}\right)\right| m_{S}\left(d b_{1}\right) \\
\leqq c_{k}(\log n)^{-\frac{1}{2}+\varepsilon} \int_{S^{1}} P_{V_{k}, V_{n}}^{S^{1}}\left(b_{1}, x_{V_{k}} \mid x_{\overline{V_{n}}}\right) m_{S}\left(d b_{1}\right) .
\end{array}
$$


It 1 s easy to derive our theorem from this lemma (when $G=S^{1}$ ). We have from (1.6), (2.1), and (1.4), that

$$
\begin{aligned}
\int_{S^{1}}\left|P^{S^{1}}\left(b_{1}, x_{V_{k}}\right)-P^{S^{1}}\left(g_{0} b_{1}, x_{V_{k}}\right)\right| m_{S}\left(d b_{1}\right) \\
\quad \leqq \int_{X \mathbb{Z}^{2}}\left[\int_{S^{1}}\left|P_{V_{k}, V_{n}}^{S^{1}}\left(b_{1}, x_{V_{k}} \mid y_{\overline{V_{n}}}\right)-P_{V_{k}, V_{n}}^{S^{1}}\left(g_{0} b_{1}, x_{V_{k}} \mid y_{\overline{V_{n}}}\right)\right| m_{S}\left(d b_{1}\right)\right] \mathscr{P}\left(d y_{\mathbb{Z}^{2}}\right) \\
\quad \leqq c_{k}(\log n)^{-1 / 2+\varepsilon} \int_{S^{1}}\left[\int_{X \mathbb{Z}^{2}} P_{V_{k}, V_{n}}^{S^{1}}\left(b_{1}, x_{V_{k}} \mid y_{\overline{V_{n}}}\right) \mathscr{P}\left(d y_{\mathbb{Z}^{2}}\right)\right] m_{S}\left(d b_{1}\right) \\
\quad \leqq c_{k}(\log n)^{-1 / 2+\varepsilon} \int_{S^{1}} P^{S^{1}}\left(b_{1}, x_{V_{k}}\right) m_{S}\left(d b_{1}\right) .
\end{aligned}
$$

The conditions $\boldsymbol{A}$ and $\boldsymbol{B}$ imply, that the function $P^{S^{1}}\left(b_{1}, x_{V_{k}}\right)$ is continuous on $S^{1}$, hence from (2.3) follows

$$
P^{S^{1}}\left(b_{1}, x_{V_{k}}\right)=P^{S^{1}}\left(g_{0} b_{1}, x_{V_{k}}\right) .
$$

This means, that $P\left(g x_{V_{k}}\right)=P\left(x_{V_{k}}\right), x_{V_{k}} \in X^{V_{k}}, g \in G$. If $J \subset \mathbb{Z}^{2}$ is finite, then there exist $k$, such that $J \subset V_{k}$. Let $\mathscr{D}\left(B_{t}, t \in J\right)$ be a cylindrical set. Let $B_{t}^{1}=B_{t}$ when $t \in J$, $B_{i}^{1}=X$ when $t \in V_{k} \backslash J$, then $\mathscr{D}\left(B_{t}, t \in J\right)=\mathscr{D}\left(B_{t}^{\prime}, t \in V_{k}\right)$ hence the theorem follows from (2.4) and (1.5).

\section{Change of Variables}

Let $d \geqq d_{0}$ be fixed, where $d_{0}$ was introduced in condition $C$ on the potential. Let $s$ be any integer. The layer with number $j$ is, by definition, the set

$$
F_{j}= \begin{cases}V_{d} & j=1 \\ V_{j d} \backslash V_{(j-1)} d & j=2, \ldots, s \\ \overline{V_{s d}} & j=s+1 .\end{cases}
$$

The cardinality $a_{j}$ of $F_{j}$ is

$$
a_{j}=(2 j d+1)^{2}-(2(j-1) d+1)^{2} \leqq 8 j d^{2} ; j=2, \ldots, s .
$$

Let $T^{s}=S^{1} \times \cdots \times S^{1}$ be an $s$-dimensional torus with fixed decomposition in a product of $s$ circles $S^{1}$. We can define an action of this torus on the space of all configurations on $V_{s d}$ by the following formula:

where

$$
\boldsymbol{b} x_{V_{\mathrm{s} d}}=\left(b_{1} x_{F_{1}}, b_{2} x_{F_{2}}, \ldots, b_{s} x_{F_{s}}\right)
$$

$$
\begin{aligned}
\boldsymbol{b} & =\left(b_{1}, \ldots, b_{s}\right) \in T^{s} ; \quad b_{i} \in S^{1} ; \\
x_{V_{s d}} & =\left(x_{F_{1}}, \ldots, x_{F_{s}}\right) \in X^{V_{s d}} .
\end{aligned}
$$

Let the contribution of the finite set $A$ to the energy of the configuration $\boldsymbol{b} x_{V_{s d}}$ under the boundary condition $x_{\overline{V_{s d}}}$ be nonzero. Then we have from condition $C$ on the potential, that $d(A) \leqq d$, hence two possibilities arise:

a) For some $j A \subset F_{j}$; then the contribution of $A$ to energy of the configuration (3.2) is determined by $x_{F_{j}}$ because of the $S^{1}$-invariance of the potential.

b) For some $j A \subset F_{j} \cup F_{j+1}$, and $A \cap F_{j} \neq \emptyset, A \cap F_{j+1} \neq \emptyset$. Then the contribution of $A$ to the energy of the configuration (3.2) is determined by $x_{F_{3}}, x_{F_{j+1}}$ and 
$\left(b_{j} b_{j+1}^{-1}\right) \in S^{1}$ (where $b_{s+1} \equiv \mathbb{1} \in S^{1}$ by definition). So, we can rewrite the energy of $\boldsymbol{b} x_{V_{s d}}$ under the boundary condition $x_{\overline{V_{s d}}}$ in the following way

$$
\begin{aligned}
U\left(\boldsymbol{b} x_{V_{s d}} \mid x_{\overline{V_{s d}}}\right)= & U\left[\left(b_{1}, \ldots, b_{s}\right)\left(x_{F_{1}}, \ldots, x_{F_{s}}\right) \mid x_{\overline{V_{s d}}}\right] \\
= & \sum_{j=1}^{s} \sum_{A \subset F_{j}} U_{A}\left[\left.\left(b_{j} x_{F_{j}}\right)\right|_{A}\right] \\
& +\sum_{j=1}^{s} \sum_{\substack{A \cap F_{j} \neq \vartheta \\
A \cap F_{j+1} \neq \vartheta}} U_{A}\left[\left.\left(b_{j} x_{F_{j}}, b_{j+1} x_{F_{j+1}}\right)\right|_{A}\right] \\
= & \sum_{j=1}^{s} U_{j}\left(x_{F_{j}}\right)+\sum_{j=1}^{s} R_{j}\left(b_{j} b_{j+1}^{-1} x_{F_{j}}, x_{F_{J+1}}\right),
\end{aligned}
$$

where $U_{j}$ and $R_{j}$ denote internal sums in the first and second summand correspondingly. Let us change the coordinites $\boldsymbol{b}$ on $T^{s}$ into a new one $\boldsymbol{h}=\left(h_{1}, \ldots, h_{s}\right)$ according to the formula

$$
\left\{\begin{array}{l}
h_{j}=b_{j} b_{j+1}^{-1} \quad j=1, \ldots, s-1 \\
h_{s}=b_{s}
\end{array}\right.
$$

It's easy to see, that

$$
\frac{\partial^{2}}{\partial h_{j}^{2}} R_{j}^{S^{1}}\left(h_{j}, x_{F_{j}}, x_{F_{j+1}}\right) \leqq c_{1} j,
$$

where $c_{1}=c_{1}(d, L)$. Indeed, every summand of $R_{j}^{S^{1}}\left(h_{j}, x_{F_{j}}, x_{F_{j+1}}\right)$ has the form $U_{A}^{S^{1}}\left[h_{j},\left.x_{F_{j}}\right|_{A \cap F_{j}},\left.x_{F_{j+1}}\right|_{A \cap F_{j+1}}\right]$. But $\partial / \partial h_{j}^{2} U_{A}^{S^{1}}\left[h_{j},\left.x_{F_{j}}\right|_{A \cap F_{j}},\left.x_{F_{J+1}}\right|_{A \cap F_{j+1}}\right]$ is a sum of less than $|A|^{2} \leqq d^{4}$ summands, each of them bounded by $L$ according to the condition $\boldsymbol{B}$ on the potential. According to (3.1) the sum $R_{j}$ contains less than $c_{2} j$ summands, where $c_{2}<\infty$ depends only on $d$, hence the inequality follows.

Let's consider a set $\mathfrak{N}$ of variables $\left(\boldsymbol{b}, x_{V_{s d}} \mid x_{\overline{V_{s d}}}\right)$, where $\boldsymbol{b}=\left(b_{1}, \ldots, b_{s}\right) \in T^{s}$, $x_{V_{s d}}=\left(x_{F_{1}}, \ldots, x_{F_{s}}\right) \in X^{V_{s d}}$ and $x_{\overline{V_{s d}}}$ is fixed. We'll define a probability distribution on $\mathfrak{N}$ by the Gibbs density:

$$
\begin{aligned}
p\left(\boldsymbol{b}, x_{V_{s d}} \mid x_{\overline{V_{s d}}}\right)=p\left(\left(b_{1}, \ldots, b_{s}\right),\left(x_{F_{1}}, \ldots, x_{F_{s}}\right) \mid x_{\overline{V_{s d}}}\right) \\
=\frac{\exp \left\{-U^{T^{s}}\left(b_{1}, x_{F_{1}}, \ldots, b_{s}, x_{F_{s}} \mid x_{\overline{V_{s d}}}\right)\right\}}{\int_{T^{s}}\left(\int_{X^{V_{s d}}} \exp \left\{-U^{T^{s}}\left(b_{1}, x_{F_{1}}, \ldots, b_{s}, x_{F_{s}} \mid x_{\overline{V_{s d}}}\right)\right\} \mu_{V_{s d}}\left(d x_{V_{s d}}\right)\right) m_{T}(d \boldsymbol{b})} .
\end{aligned}
$$

(We suppose here, that $m_{T}\left(T^{s}\right)=1$.) Let's put in (2.2) $k=d, n=s d$, then $V_{k}=F_{1}$, $\overline{V_{n}}=\overline{V_{s_{d}}}$. Dividing both sides in the inequality (2.2) by the integral in the right-hand side, we get the following inequality:

$$
\int_{S^{1}}\left|p\left(b_{1} \mid x_{F_{1}}, x_{\overline{V_{s d}}}\right)-p\left(g_{0} b_{1} \mid x_{F_{1}}, x_{\overline{V_{s d}}}\right)\right| m_{S}\left(d b_{1}\right) \leqq c_{k}(\log s d)^{-1 / 2+\varepsilon}
$$

where the conditional density $p\left(b_{1} \mid x_{F_{1}}, x_{\overline{V_{s d}}}\right)$ is computed in $\mathfrak{M}$. It's more convenient to study conditional density $p\left(b_{1} \mid x_{F_{1}}, \ldots, x_{F_{s}}, x_{\overrightarrow{V_{s d}}}\right)$. The connection between them is well-known:

$$
\begin{aligned}
& p\left(b_{1} \mid x_{F_{1}}, x_{\overline{V_{s d}}}\right) \\
& \quad=\int p\left(b_{1} \mid x_{F_{1}}, \ldots, x_{F_{s}}, x_{\overline{V_{s d}}}\right) p\left(x_{F_{2}}, \ldots, x_{F_{s}} \mid x_{F_{1}}, x_{\overline{V_{s d}}}\right) \mu_{V_{s d} \backslash F_{1}}\left(d x_{F_{2}} \ldots d x_{F_{s}}\right) .
\end{aligned}
$$


We have

$$
\begin{aligned}
& p\left(\boldsymbol{b} \mid x_{V_{s d}}, x_{\overline{V_{s d}}}\right)=\frac{\exp \left\{-U^{T^{s}}\left(\boldsymbol{b}, x_{V_{s d}} \mid x_{\overline{V_{s d}}}\right)\right\}}{\int_{T^{s}} \exp \left\{-U^{T^{s}}\left(\boldsymbol{b}, x_{V_{s d}} \mid x_{\overline{V_{s d}}}\right)\right\} m_{T}(d \boldsymbol{b})} \\
= & p\left(\boldsymbol{h} \mid x_{V_{s d}}, x_{\overline{V_{s d}}}\right)=\prod_{j=1}^{s} p_{j}\left(h_{j} \mid x_{F_{J}}, x_{F_{J+1}}\right)
\end{aligned}
$$

where

$$
p_{j}\left(h_{j} \mid x_{F_{j}}, x_{F_{j}+1}\right)=\frac{\exp \left\{-R_{j}^{S^{1}}\left(h_{j}, x_{F_{j}}, x_{F_{j+1}}\right)\right\}}{\int_{S^{1}} \exp \left\{-R_{j}^{S^{1}}\left(h_{j}, x_{F_{j}}, x_{F_{j+1}}\right)\right\} m_{S}\left(d h_{j}\right)} .
$$

The last equalities follow from (3.3) and because the fact that the Jacobian of $\boldsymbol{b} \rightarrow \boldsymbol{h}$ is equal to 1 . We have from (3.7) that the elements $h_{j}$ are independent, and it is easy to see, that $h_{1} \ldots h_{s}=\left(b_{1} b_{2}^{-1}\right)\left(b_{2} b_{3}^{-1}\right) \ldots\left(b_{s-1} b_{s}^{-1}\right)\left(b_{s}\right)=b_{1} \in S^{1}$. The probability density of this product is a function on $S^{1}$ :

$$
\begin{gathered}
p\left(b_{1} \mid x_{V_{s d}}, x_{\overline{V_{s d}}}\right)=\left[p_{1}\left(\cdot \mid x_{F_{1}}, x_{F_{2}}\right) \circ p_{2}\left(\cdot \mid x_{F_{2}}, x_{F_{3}}\right) \circ \ldots\right. \\
\left.\cdots \circ p_{s}\left(\cdot \mid x_{F_{s}}, x_{F_{s}+1}\right)\right]\left(b_{1}\right),
\end{gathered}
$$

where $\circ$ means convolution on $S^{1}$, i.e.

$$
\left(q_{1} \circ q_{2}\right)(x)=\int_{S^{1}} q_{1}(y) q_{2}\left(x y^{-1}\right) m_{s}(d y)=\sum_{r \in \mathbb{Z}} q_{1} * q_{2}(x+r)
$$

in the last equality we consider real-valued functions, obtained by identification of $S^{1}$ and a segment $[0,1]$.

\section{Proof of the Main Result}

It remains to prove the following

Lemma 2. For any $\varepsilon>0$ and $d \geqq d_{0}$ there is a constant $c>0$, such that for any $s \geqq 1$, any $x_{V_{s d}}$ and any $x_{\overline{V_{s d}}}$,

$$
\int_{S^{1}}\left|p\left(b_{1} \mid x_{V_{s d}}, x_{\overline{V_{s d}}}\right)-1\right| m_{S}\left(d b_{1}\right) \leqq c(\log s)^{-1 / 2+\varepsilon} .
$$

Proof. Let $v_{j} \in S^{1}$ be such, that

$$
R_{j}^{S^{1}}\left(v_{j}, x_{F_{j}}, x_{F_{J+1}}\right)=\min _{h \in S^{1}} R_{j}^{S^{1}}\left(h, x_{F_{j}}, x_{F_{j+1}}\right) .
$$

Then

$$
\left.\frac{\partial R_{j}^{S^{1}}}{\partial h}\left(h, x_{F_{J}}, x_{F_{j+1}}\right)\right|_{h=v_{j}}=0
$$

We'll use the same notation $R_{j}^{S^{1}}$ for the corresponding real-valued function on $[0,1]$. Using Taylor's formula in the Lagrange form and the estimation (3.4) we have

$$
R_{j}^{S^{1}}\left(h, x_{F_{j}}, x_{F_{j+1}}\right) \leqq R_{j}^{S^{1}}\left(v_{j}, x_{F_{j}}, x_{F_{j+1}}\right)+\frac{c_{1} j}{2}\left|h-v_{j}\right|^{2}
$$


Then we have

$$
\begin{aligned}
& \int_{S^{1}} \exp \left\{-R_{j}^{S^{1}}\left(h, x_{F_{j}}, x_{F_{j+1}}\right)\right\} m_{S}(d h) \geqq \exp \left\{-R_{j}^{S^{1}}\left(v_{j}, x_{F_{j}}, x_{F_{j+1}}\right)\right\} \\
& \times \int_{0}^{1} \exp \left\{-\frac{1}{2} c_{1} j\left|y-v_{j}\right|^{2}\right\} d y \geqq c_{3}^{-1} \exp \left\{-R_{j}^{S^{1}}\left(v_{j}, x_{F_{j}}, x_{F_{j+1}}\right)\right\} j^{-1 / 2}
\end{aligned}
$$

where $c_{3}=c_{3}(d, L)$. From (3.8) we have that

$$
p_{j}\left(h_{j} \mid x_{F_{J}}, x_{F_{J}+1}\right) \leqq c_{3} j^{1 / 2} ; \quad j=1, \ldots, s .
$$

Let us consider the convolution

$$
p^{*}\left(\cdot \mid x_{V_{s d}}, x_{\overline{V_{s d}}}\right)=p_{1}\left(\cdot \mid x_{F_{1}}, x_{F_{2}}\right) * \cdots * p_{s}\left(\cdot \mid x_{F_{s}}, x_{F_{s+1}}\right)
$$

where the right-hand side real-valued functions are obtained by identification of $S^{1}$ with $[0,1]$. For estimation of this convolution we make use of a local limit theorem of Statulevicius [13] for non identically distributed random variables.

Let

$$
E\left(x_{F_{J}}, x_{F_{J+1}}\right)=\int_{0}^{1} y p_{j}\left(y \mid x_{F_{J}}, x_{F_{J+1}}\right) d y .
$$

Then, from (4.2) it follows the existence of a constant $c_{4}=c_{4}(d, L)$, such that the conditional variance of $h_{j}$ (as of real-valued random variable) is

$$
D\left(x_{F_{J}}, x_{F_{J+1}}\right)=\int_{0}^{1}\left(y-E\left(x_{F_{J}}, x_{F_{J+1}}\right)\right)^{2} p_{j}\left(y \mid x_{F_{J}}, x_{F_{J+1}}\right) d y \geqq c_{4} j^{-1} .
$$

[It is easy to see, that among the distributions, satisfying (4.2), the distribution

$$
p(y)= \begin{cases}c_{3} j^{1 / 2} & 0 \leqq y \leqq c_{3}^{-1} j^{-1 / 2} \\ 0 & y>c_{3}^{-1} j^{-1 / 2}\end{cases}
$$

has the smallest variance.]

But the sum $\Sigma j^{-1}$ diverges, so we can apply the local limit theorem of [13] to the sequence $h_{j}$. This theorem guarantees us, that our density $p^{*}$ is near to the Gaussian density. We'll get precise estimations after substitution of the formula (4.2) of this work into the formulas (3.1), (3.2) of [13]. Then for

$$
E_{s}=\sum_{j=1}^{s} E\left(x_{F_{J}}, x_{F_{J+1}}\right), D_{s}=\sum_{j=1}^{s} D\left(x_{F_{J}}, x_{F_{J+1}}\right),
$$

and for some $c_{5}=c_{5}(d, L)$ we have

$$
\int_{-\infty}^{+\infty}\left|p^{*}\left(y \mid x_{V_{s d}}, x_{\overline{V_{s d}}}\right)-\left(2 \pi D_{s}\right)^{-1 / 2} \exp \left\{-\left(2 D_{s}\right)^{-1}\left(y-E_{s}\right)^{2}\right\}\right| d y \leqq c_{5}(\log s)^{-1 / 2+\varepsilon} .
$$

By definition of the convolution on $S^{1}$ [see formula (3.9)] we have

$$
p\left(\cdot \mid x_{V_{s d}}, x_{\overline{V_{s d}}}\right)=\sum_{r \in \mathbb{Z}} p^{*}\left(\cdot+r \mid x_{V_{s d}}, x_{\overline{V_{s d}}}\right) .
$$


Finally, there exists $c_{6}=$ const, such that for any $D_{s}>1$

$$
\left|\sum_{r \in \mathbb{Z}}\left[\left(2 \pi D_{s}\right)^{-1 / 2} \exp \left\{-\left(2 D_{s}\right)^{-1}\left(y-r+E_{s}\right)^{2}\right\}\right]-1\right| \leqq c_{6}\left(D_{s}\right)^{-1 / 2} .
$$

Hence, Lemma 2 follows.

Lemma 1 is equivalent to the inequality (3.5), which follows immediately from (4.1) and (3.6).

\section{Proof in the General Case}

The proof in the general case is based on well known results on Lie groups $[4,5]$. Every compact connected Lie group $G$ is a complete Riemanian manifold. So we can join every element of $G$ with $\mathbb{1} \in G$ by geodesical curve. The minimal algebraic subgroup of $G$, which contain this curve, can be closed or not. In the first case this subgroup is isomorphic to $S^{1}$, in the second - to $\mathbb{R}^{1}$. But the group $\mathbb{R}^{1}$ is commutative, so its closure in $G$ is also a (compact) commutative Lie subgroup of $G$. But every compact commutative Lie group is a torus, hence every element of $G$ belongs to some torus $T^{k} C G$. Next, every element of $T^{k}$ is a product of no more than $k$ elements, each of them belonging to some compact one-dimensional subgroup. Hence, every element of a compact connected Lie group of dimension $n$ can be represented as a product of no more than $n$ elements of several compact one-dimensional subgroups of $G$.

So, it is enough to verify our theorem for every compact one-dimensional subgroup. But conditions $\boldsymbol{A}-\boldsymbol{D}$ on the potential $U$ and the measure $\mu$ can be restricted to every compact one-dimensional subgroup. We must only verify that the restriction of every function of class $\mathscr{C}^{2}$ according to some smooth structure on $G$ on every compact one-dimensional subgroup of $G$, remains again in the class $\mathscr{C}^{2}$ according to standard smooth structure on $S^{1}$. The proof of this fact is in $[4]$.

\section{Discussion}

The following example explains the importance of our assumptions about the existence of second derivatives of the potential and two-dimensionality of lattice. Suppose $X=G=S^{1}, d=d_{0}=1$, and the potential $U=\left\{U_{A}\right\}$ is

$U_{\{s, t\}}\left(x_{s}, x_{t}\right)=\left\{\begin{array}{l}\infty ; s, t \text { belong to the same layer, } x_{s} \neq x_{t} ; \\ 0 ; s, t \text { belong to the same layer } x_{s}=x_{t} ; \\ f\left(\left|x_{s}-x_{t}\right|\right) ;|s-t|=1, s, t \text { belong to neighbouring layers, }\end{array}\right.$

where $f$ is any function, and finally, $U_{A} \equiv 0$ for any other $A$. In this model all the spins of the same layer take the same value, and the differences $y_{j}=x_{j} \cdot x_{j+1}^{-1}$ are independent. $y_{j}$ has a probability distribution with density

$$
p_{j}\left(y_{j}\right)=\frac{\exp \left\{-f\left(\left|y_{j}\right|\right) \varphi_{j}\right\}}{\int_{X} \exp \left\{-f\left(\left|y_{j}\right|\right) \varphi_{j}\right\} \mu\left(d y_{j}\right)}
$$


where $\varphi_{j} \sim 4 j$ is the number of neighbouring points in neighbouring layers. If $f(y)$ is continuous, $f(|y|)<f(0)$ for any $|y|$ and $f(|y|)-f(0) \sim-k|y|^{\alpha}$ for $|y| \rightarrow 0$, where $\alpha>0, k>0$, then we have from (6.1) the variance $D\left(y_{i}\right)$ is of order $j^{-2 / \alpha}$ when $j \rightarrow \infty$. Hence the sum $\Sigma D\left(y_{j}\right)$ diverges for $\alpha \geqq 2$ and converges for $\alpha<2$. Hence, by the above mentioned limit theorems, under any boundary condition $x_{\overline{V_{s}}}$, the distribution of $b_{1}$ tends to the uniform distribution when $s \rightarrow \infty$ and $\alpha \geqq 2$, and does not tend to it, when $\alpha<2$. In $v$-dimensional case $\varphi_{j}$ is of order $j^{\nu-1}$, and the same arguments give that the distribution of $b_{1}$ tends to the uniform one, when $\alpha \geqq 2(v-1)$ and does not tend to it, if $\alpha<2(v-1)$. So, when $v=3$, we'll have a uniform distribution for $b_{1}$ only if in the minimum of the potential the second and third derivatives are zeroes.

In this model we have less fluctuations, than in the case of translation-invariant potential. So, if there is no break-down of continuous symmetry in this model, then the same situation is in general case. Our main idea was to reduce the general case by introducing some conditions to a situation, similar to the model of this section. It is clear, that we cannot reverse our arguments. Indeed, it is possible to deduce from [14] that in translation-invariant case the Gibbs state is unique and there is no break-down of continuous symmetry for any $\alpha$, if the temperature is high enough. It seems, that there is break-down of symmetry, when $\alpha<2(v-1)$ and the temperature is low enough, but still we have no proof.

\section{References}

1. Ruelle, D.: Statistical Mechanics. Rigorous results. New York-Amsterdam: W. A. Benjamin Inc. 1969

2. Gallavotti, G. : Riv. Nuovo Cim. 2, $133-169$ (1972)

3. Bortz, A. B., Griffiths, R. B.: Commun. math. Phys. 26, 102-108 (1972)

4. Pontriagin, L.S.: Topological groups (in Russian); 3-rd edition, 1973, Moscow Science

5. Chevalley, C.: Theory of Lie-groups. Vol. 1, Princeton: University Press 1946

6. Dobrushin, R. L. : Funct. Anal. Appl. (in Russian) 2, $31-43$ (1968)

7. Dobrushin, R. L.: Funct. Anyl. Appl. (in Russian). 3, 27-35 (1969)

8. Lanford,O.E., Ruelle, D.: Commun. math. Phys. 13, 194-215 (1968)

9. Mermin, N. D.: J. Math. Phys. 8, 1061-1064 (1967)

10. Fisher, M.E., Jasnov, D.: Phys. Rev., B, Solid States 3, 907-924 (1971)

11. Garrison, I.C., Wong, I., Morrison, H.L.: Math. Phys. 13, 1735-1742 (1972)

12. Fisher, M.E.: J. Appl. Phys. 38, 981-990 (1967)

13. Statulevičius, V.A.: Theory Probability Appl. (in Russian) 10, 645-659 (1965)

14. Dobrushin, R. L.: Theory probability Appl. (in Russian.) 15, 169-497 (1970) 